\title{
ARCHoN: Adaptive Range Control of Hotzone Cells in Heterogeneous Cellular Networks
}

\author{
Ji-Hoon Yun \\ Department of Electrical and Information Eng. \\ Seoul National University of Science and Technology \\ 232 Gongneung-ro, Nowon-gu, Seoul, Korea \\ Email: jhyun@seoultech.ac.kr
}

\author{
Kang G. Shin \\ Real-Time Computing Laboratory, EECS Department \\ The University of Michigan \\ Ann Arbor, MI 48109-2121, U.S.A. \\ Email: kgshin@eecs.umich.edu
}

\begin{abstract}
Heterogeneous networks (HetNets) are typified by cellular deployments with multiple types of cells of different sizes and overlapping coverage areas using a common frequency band. Especially, hotzone cells overlaid on a macrocell to cover hotspot areas are expected to prevail in HetNets, thus cost-effectively enhancing cellular capacity via spatial reuse of spectrum resource and offloading macrocells. In order to fully achieve such benefits, users need to be properly distributed/assigned to the overlaid hotzone cells such that the radio resources therein are fully utilized. To this end, we propose a new architecture called Adaptive Range Control of Hotzone Cells for Heterogeneous Networks (ARCHoN) that jointly controls the radio resource allocations and ranges of OFDMA-based hotzone cells. The use of cell ranges for distributing users in $\mathrm{ARCHoN}$ is advantageous in that it can be implemented within a conventional cell-selection framework without modifying user devices or an air interface. In ARCHoN, each cell allocates users radio (frequency, time and power) resources in a non-cooperative manner, deriving a sequence of allocations monotonically decreasing the entire load. For range control, two algorithms are proposed: per-cell and universal, which have a tradeoff between performance and computational complexity. The solution yielded by the combination of these radio resource and range control algorithms is analytically proven to converge to a unique fixed point. Our indepth evaluation has shown ARCHoN to significantly improve the service quality of users; in an example simulation scenario, ARCHoN is shown to improve the signal-to-interference and noise ratios (SINRs) of users, on average, by up to $3.5 \mathrm{~dB}$ in downlink and $18.8 \mathrm{~dB}$ in uplink, over the case of the conventional handover framework.
\end{abstract}

\section{INTRODUCTION}

Rapidly growing demands for bandwidth-hungry data services in wireless cellular networks have been a driving force to enhance system capacity in general, and develop spectrally efficient physical-layer solutions in particular. However, advanced air interfaces using such solutions alone may not be able to meet this rapidly growing need for wireless network capacity. Increasing the number and the density of macrocells and expanding their service-bandwidth by licensing additional frequency spectra can be considered in parallel, but the associated cost could be too high to be economically feasible.

Heterogeneous networks (HetNets) have emerged as a costeffective means to enhance cellular coverage and capacity [1]; for example, the $3 \mathrm{G}$ Partnership Project (3GPP) has recently added HetNets to the scope of the LTE-Advanced Study [2]. HetNets represent cellular deployments with heterogeneous types of cells of different sizes and overlapping coverage areas. Especially, medium-to-small-sized cells, called hotzone cells, are gaining considerable attention as a cost-efficient solution to handle non-uniform traffic distributions and expected to prevail in HetNets. In general, user demands are not geographically uniform, i.e., there may exist small hotspot areas where traffic demands are higher than other areas due to high population density (e.g., shopping malls, train stations). Although a macrocell's connectivity may already be available there, existing macrocells could have insufficient capacity and get overloaded easily. Adding another macrocell to cover such small areas exceeds the requirement and hence wastes the operator's capital investment. Hotzone cells are dedicated to this deployment scenario (covering small hotspot areas to provide sufficient capacity).

Due to the high cost of licensed spectrum, operators may allocate hotzone cells the same frequency band as macrocells, called co-channel deployment. Hotzone cells with same channels allocated as the macrocell are overlaid on the macrocell and spatially reuse the macrocell's channels, thus greatly enhancing the total capacity of a cellular network. Besides, since a large amount of user traffic will be served by local hotzone cells in HetNets, the macrocell's traffic will be offloaded to the hotzone cells, reducing/eliminating the need for costly upgrade of macro base stations (BSs).

One of the main challenges associated with HetNets is how to distribute users between macro and hotzone cells while mitigating the accompanying co-channel interferences. In HetNets, due to the overlapping deployment of cells, it is common for users to have multiple candidate cells to be connected to, not only near cell boundaries but also in inner areas. However, the conventional strongest-receivingpower-based cell selection is not optimal in this deployment scenario. For example, although a user receives a higherpower signal from a macrocell (due to a stronger transmit power) than from a closer hotzone cell, he could experience better service quality when served by the hotzone cell if the hotzone cell is less loaded and thus can allocate him sufficient radio resources (frequency, time or power) while the macrocell cannot. Such a situation is likely to happen since, due to a smaller coverage area, a hotzone cell typically serves less users than a macrocell does. Moreover, users may experience 
and produce fewer interferences when served by local hotzone cells due to reduced path losses. Therefore, users' serving cells in HetNets should be determined by considering the load and interference conditions as well as the signal strengths of candidate cells.

To overcome the inefficiency of the conventional cell selection in HetNets, range extension, which expands the service ranges of overlaid hotzone cells by adjusting cell-selectionrelated parameters and thus let them serve more users, is under discussion for LTE-Advanced [3]. Here, a cell's range is defined as an area within which users get connected to the cell. The performance gain of hotzone cells' range extension has already been reported in several 3GPP contribution proposals [4]. However, detailed algorithms determining ranges are beyond the scope of specifications and remain vendoror operator-specific. Within the framework of existing cellular systems, a cell's range can be controlled by adjusting a per-cell offset to the received signal strength of the cell's pilot signal which is used in a handover-decision procedure. Therefore, the target of a range-control algorithm narrows into the adjustment of these per-cell offsets of each cell for its neighbor cells.

In this paper, we propose a joint radio-resource- and range-control architecture for OFDMA-based HetNets called Adaptive $\mathbf{R}$ ange Control of $\mathbf{H}$ otzone cells in $\mathbf{H}$ eterogeneous Networks (ARCHoN). ARCHoN allocates radio resources (frequency, time and power) and distributes/assigns users among overlapping cells by controlling hotzone cells' ranges such that the overall downlink (DL) and uplink (UL) loads of a HetNet are minimized while per-user rate constraints are met. To design detailed control algorithms, we transform this load-minimization problem into an equivalent but simpler form having a smaller number of variables and identify the condition of these variables to suppress ping-pong handovers. The solution yielded by ARCHoN is guaranteed to converge to a unique fixed point. ARCHoN is designed to operate in a cell-selection framework of existing cellular systems, without requiring the modification of user devices or an air interface. To the best of our knowledge, ARCHoN is the first architecture jointly controlling the resource allocation and ranges of hotzone cells in the literature.

ARCHoN is composed of three complementary controllers: (1) master, (2) radio resource, and (3) range. The master controller is in charge of activating and deactivating the range controller according to cells' conditions reported by the radio resource controller of each cell via inter-cell signaling interfaces; to reduce the signaling overhead, a range control is triggered only when absolutely needed and on limited cells. The radio resource controller of each cell allocates users radio resources in a non-cooperative manner; it derives a sequence of allocations monotonically decreasing the load of its own cell and finally reaching a Nash equilibrium. For the operation of the range controller, we first develop a per-cell range-control algorithm, which iteratively determines cell-specific offset parameters and is applicable to all cell structure scenarios of HetNets. Then, for UL-resource-limited HetNets, we propose the universal control algorithm that immediately maps cells' conditions to per-cell offsets with the introduction of a single network-wide control parameter. Thus, it has a reduced search space with no dependency on the number of cells, lowering computational complexity and signaling overhead compared to the per-cell control, and can be implemented in a distributed manner, but at the expense of degraded performance.

Our evaluation results show that $\mathrm{ARCHoN}$ improves the performance of users served by both macro and hotzone cells in various environmental settings. In an example scenario with 30 users and 20 hotzone cells within a macrocell, users achieve higher signal-to-interference and noise ratios (SINRs) than the case without ARCHoN, on average, by $3.5 \mathrm{~dB}$ (per-cell control) and $2.7 \mathrm{~dB}$ (universal control) in DL, and by $18.8 \mathrm{~dB}$ (per-cell control) and $9.7 \mathrm{~dB}$ (universal control) in UL.

The rest of this paper is organized as follows. Sections II and III describe the related work and the system model, respectively. Section IV presents the ARCHoN architecture, and Section V describes the control algorithms. Section VI evaluates ARCHoN via detailed simulations, and Section VII concludes the paper.

\section{RELATED WORK}

Range extension of hotzone cells has been studied and is still under discussion in 3GPP working groups for LTEAdvanced, but the studies mostly focus on evaluating the performance gain of the concept and exploring potential impacts on specifications [3][4]. To realize the benefit of the range extension, vendors need an algorithm to control the cell ranges, which is one of the contributions of this paper. There has been an attempt to control access points' ranges in 802.11 WLANs by adjusting the transmit powers of beacons [5]. However, this work assumes no power control of data channels and fixed load contribution of a user on an access point. Thus, it is not readily applicable to cellular systems. Moreover, this beacon-power adjustment approach cannot support user-condition-dependent range control. ${ }^{1}$

Researches on radio resource assignment in OFDMA-based systems are also relevant to our work. Many of them focused on finding an assignment of frequency (subcarrier) and power resources for sum-rate maximization in a single cell [6][7]. Some recent work considered the multi-cell case based on cells' non-cooperative operations [8][9] or inter-cellular cooperations [10]. These researches assume that the user set of each cell is given and fixed.

There have been numerous proposals to solve the BS assignment problem on a per-user basis, i.e., each user makes a decision which cell to connect, mostly for CDMA systems. Yates and Huang [11] proposed distributed algorithms that find the optimal UL power vector and BS assignment. Lee et al. [12] developed a pricing-based BS assignment algorithm considering the congestion level of the BS. Iterative algorithms based on linear programming (LP) were presented in [13]. Kim et al. [14] focused on flow-level cell load balancing. However,

\footnotetext{
${ }^{1}$ The offset adjustment is more beneficial than changing the transmit power of a pilot signal in the sense that different offsets can be applied according to the users' conditions, such as movement speed.
} 
these per-user association approaches require user devices to be modified for inclusion of a new decision algorithm and possibly new signaling with BSs over the air. Therefore, they can be realized via a new standard and the corresponding new user devices only.

\section{System MODEL}

This section describes the network architecture under consideration. A cell-selection procedure assumed in this paper is described and a load metric is defined as well.

\section{A. Assumptions}

We consider a typical two-layer HetNet architecture in which hotzone cells are overlaid on a macrocell. The macrocell (indexed as cell 0 ) and the set of hotzone cells $\mathcal{H}=$ $\{1, \ldots, C-1\}$ are assumed to use an identical radio access technology (RAT) based on OFDMA and a common frequency band; we then denote the set of all cells by $\mathcal{C}(=\{0\} \cup \mathcal{H})$. Cell $i$ operates under the control of BS $i$. The neighbor cells of cell $i$ are denoted by $N_{i}$. The set of users $U$ is divided into the set $U_{i}$ of users being served by cell $i \in \mathcal{C}$. We also assume that neighbor cells establish inter-cell signaling interfaces over wired networks as, for example, the X2 interface in LTE [15]. The frequency band is composed of multiple resource blocks (RBs), each of which is a group of contiguous subcarriers and also the minimum scheduling granularity, and the set of given RBs is denoted by $\mathcal{K}$. We assume that a user's resource usage is evenly distributed in frequency and time domains (like RBs of distributed type in LTE [16]) to randomize intercell interference so that significant performance degradation at a certain frequency resource or time slot is avoided. Similar assumptions have also been adopted in previous work [14].

\section{B. Cell-Selection Framework}

We assume a typical cell-selection mechanism used in general cellular systems (e.g., LTE-Advanced [17]). Let $\theta_{S(j)}$ and $\theta_{T(j)}$ be the pilot transmit powers of the serving cell and a target cell of user $j$, respectively; $h_{i, j}(\leq 1)$ be the channel gain from cell $i$ to user $j$. A user $j$ 's cell change is triggered by his serving cell if, in the $\mathrm{dB}$ scale,

$$
\theta_{S(j)}^{(\mathrm{dBm})}+h_{S(j), j}^{(\mathrm{dB})}<\theta_{T(j)}^{(\mathrm{dBm})}+h_{T(j), j}^{(\mathrm{dB})}-\lambda_{S(j), T(j)}^{(\mathrm{dB})}
$$

where $\lambda_{S(j), T(j)}$ is an offset parameter of the target cell $\left(\lambda_{S(j), T(j)} \in \Lambda ; \Lambda\right.$ is a discrete set). ${ }^{2}$ Therefore, a cell's range is adjusted by its offset parameter; a larger offset leads to a smaller range of the cell. Provided that the set of users served by cell $i$ is given as $U_{i}$ and a vector of offset values of its neighbor cells is denoted by $\vec{\lambda}_{i}$, the set of users to switch to cell $i^{\prime} \in N_{i}$ is determined by

$$
U_{i^{\prime}}\left(\vec{\lambda}_{i}\right)=\left\{j \mid i^{\prime}=\arg \max _{\left\{i, v \in N_{i}\right\}}\left(h_{i, j} \theta_{i},\left\{h_{v, j} \theta_{v} / \lambda_{i, v}\right\}\right), j \in U_{i}\right\} .
$$

We assume that the transmit powers of cells' pilot signals are fixed.

\footnotetext{
${ }^{2}$ In order to suppress frequent cell changes due to users wandering along a cell boundary or channel gain fluctuations, a hysteresis parameter can also be used.
}

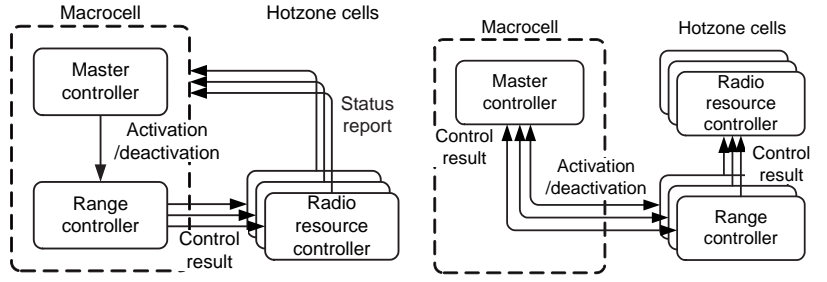

(a) Centralized range control (with (b) Distributed range control (with the per-cell range control algorithm) the universal range control algorithm)

Fig. 1. Interactions between the controllers of $\mathrm{ARCHoN}$

\section{Definition of Cell and Network Load}

Let $a_{j, k}$ and $p_{j, k}\left(b_{j, k}\right.$ and $\left.q_{j, k}\right)$ be the fraction of time and power allocated to user $j$ for RB $k$, respectively, in DL (UL), and $\vec{a}_{i}$ and $\vec{p}_{i}\left(\vec{b}_{i}\right.$ and $\left.\vec{q}_{i}\right)$ be their vectors for cell $i$. The radio resource allocation vectors of users served by cell $i$ are defined as $\vec{x}_{i} \triangleq,\left(\vec{a}_{i}, \vec{p}_{i}\right)$ and $\vec{y}_{i} \triangleq,\left(\vec{b}_{i}, \vec{q}_{i}\right)$. We then define the cell load function, denoted by ${ }^{3} \rho_{i}:\left(\Re^{|\mathcal{K}|} \times \Re^{\left|U_{i}\right|}\right) \times\left(\Re^{|\mathcal{K}|} \times\right.$ $\left.\Re^{\left|U_{i}\right|}\right) \rightarrow \Re$, that measures the usage of frequency, time and power resources of a cell; it is the sum of DL and UL loads as described below:

$$
\rho_{i}\left(\vec{x}_{i}, \vec{y}_{i}\right) \triangleq \rho_{i, D L}\left(\vec{x}_{i}\right)+\zeta_{i} \rho_{i, U L}\left(\vec{y}_{i}\right)
$$

and the DL load $\rho_{i, D L}$ is defined as

$$
\rho_{i, D L}\left(\vec{x}_{i}\right) \triangleq \sum_{j \in U_{i}} \sum_{k \in \mathcal{K}} a_{j, k}+\sum_{j \in U_{i}} \sum_{k \in \mathcal{K}} \omega_{j, k} \bar{p}_{j, k}
$$

where the first and second terms of the right-hand side are the consumption of frequency-time and power resources, respectively; $\bar{p}_{j, k}$ is the average DL transmit power; $\zeta_{i}$ and $\omega_{j, k}$ are balancing constants. The UL load $\rho_{i, U L}$ is defined similarly. We then extend the cell load function to the network load function, denoted by $\rho$, whose arguments are for the entire users of a network; it is expressed as

$$
\rho(\vec{x}, \vec{y})=\sum_{i \in \mathcal{C}} \chi_{i} \rho_{i}\left(\vec{x}_{i}, \vec{y}_{i}\right)
$$

where $\vec{x}$ and $\vec{y}$ are the resource allocation vectors of the entire users and $\chi_{i}$ is a cell-specific weighting constant. It is clear that both load functions are monotonically increasing in the argument vectors, i.e., if $\vec{x}^{\prime} \succeq \vec{x}$ and $\vec{y}^{\prime} \succeq \vec{y}, \rho\left(\vec{x}^{\prime}, \vec{y}^{\prime}\right) \geq$ $\rho(\vec{x}, \vec{y}) .^{4}$

\section{The ARCHoN ARCHITECTURE}

We first present the architecture of ARCHoN, its basic concept and design rationale. Then, we formulate the problems for the design of control algorithms for ARCHoN.

\footnotetext{
${ }^{3}|\cdot|$ is the cardinality of a set.

${ }^{4}$ The curled inequality symbol $\succeq$ and its strict form $\succ$ represent componentwise inequality.
} 


\section{A. Overview of ARCHoN}

$\mathrm{ARCHoN}$ is composed of three interacting decision-making controllers as illustrated in Fig. 1. The master controller decides on activating/deactivating the range controller such that a network is kept within a desirable range of load with the reduced rate of inter-cell signaling; a decision is made based on the current status of radio resource usage reported by the radio resource controller of each cell (with per-cell range control) or based on the status indirectly obtained from the control result of the range controller of each cell (with universal range control; the details will be described in Section V.C), i.e., activation when the network load exceeds a certain threshold (activation threshold) and deactivation if the network load gets lower than a certain point (deactivation threshold). The range controller, once activated, finds a range sequence to reach a target system condition iteratively; in the centralized case (Fig. 1(a)), it then informs cells of the result. Meanwhile, the radio resource controller of each cell schedules frequency, time and power resources at regular intervals to meet per-user rate requirements of connected users that are determined by the range controller.

\section{B. Problem Formulation}

Let $\vec{\lambda}$ be the vector of $\lambda_{i_{1}, i_{2}}$ for all pairs $\left(i_{1}, i_{2}\right)$ of neighboring cells. Then, ARCHoN aims to find $(\vec{x}, \vec{y}, \vec{\lambda})$ that minimizes the load of the entire network while meeting users' rate requirements in both DL and UL. Resource allocation done by the radio resource controller will be influenced by $\vec{\lambda}$. We denote this dependency of $\vec{x}$ and $\vec{y}$ on $\vec{\lambda}$ by $\vec{x}(\vec{\lambda})$ and $\vec{y}(\vec{\lambda})$. Therefore, the load minimization problem can be defined as follows.

Definition 1 (Load minimization problem):

$$
\begin{aligned}
P 1: & \min _{(\vec{x}(\vec{\lambda}), \vec{y}(\vec{\lambda}), \vec{\lambda})} \rho(\vec{x}(\vec{\lambda}), \vec{y}(\vec{\lambda})) \\
\text { subject to } & \\
C 1.1: & 0 \leq a_{j, k}, b_{j, k} \leq 1, \sum_{j \in U_{i}} a_{j, k}, \sum_{j \in U_{i}} b_{j, k} \leq 1 \\
C 1.2: & : 0 \leq p_{j, k} \leq P_{\max }, 0 \leq q_{j, k} \leq Q_{\max } \\
C 1.3: & \sum_{k \in \mathcal{K}} a_{j, k} B \log _{2}\left(1+\gamma_{j} / \Omega\right)=r_{j} \\
& \sum_{k \in \mathcal{K}} b_{j, k} B \log _{2}\left(1+\delta_{j} / \Omega\right)=s_{j} \\
C 1.4: \vec{\lambda} \in \Lambda^{C} &
\end{aligned}
$$

where $P_{\max }$ and $Q_{\max }$ are the maximum allowable transmit powers at a RB for DL and UL, respectively; $\gamma_{j}$ and $\delta_{j}$ are the target SINRs, and $r_{j}$ and $s_{j}$ are the data rates requested by user $j$ for DL and UL, respectively; $\Omega=-\ln (5 \mathrm{BER}) / 1.6$ is a constant SNR gap for the required bit error rate (BER) [18]; $B$ is the bandwidth of a RB.

In what follows, we make two important observations on the structure of this problem.

Proposition 1: To avoid a ping-pong type of cell changes, $\lambda_{i_{1}, i_{2}} \lambda_{i_{2}, i_{1}} \geq 1$ should hold for all pairs of $i_{1}, i_{2} \in \mathcal{C}$.

Proof: Suppose $i_{2}=\arg \max _{i \backslash\left\{i_{1}\right\}}\left(\theta_{i} h_{i, j} / \lambda_{i_{1}, i}\right)$. Then, user $j$ 's serving cell is changed from $i_{1}$ to $i_{2}$ if $\theta_{i_{1}} h_{i_{1}, j}<$ $\theta_{i_{2}} h_{i_{2}, j} / \lambda_{i_{1}, i_{2}}$. In order to avoid user $j$ 's immediate cell change back to $i_{1}$, the condition $\theta_{i_{2}} h_{i_{2}, j}>\theta_{i_{1}} h_{i_{1}, j} / \lambda_{i_{2}, i_{1}}$ should hold; this condition will be met if and only if $\lambda_{i_{1}, i_{2}} \geq$ $\lambda_{i_{2}, i_{1}}^{-1}$.

Proposition 2: The previous cell-selection rule can be transformed into the one having cell-wide offset parameters:

$$
U_{i}(\vec{\lambda})=\left\{j \mid i=\arg \max _{i}\left(h_{i, j} \theta_{i} / \lambda_{i}\right), j \in U\right\}
$$

if $\lambda_{i_{1}, i_{2}}$ is mapped to $\frac{\lambda_{i_{2}}}{\lambda_{i_{1}}}$ for all pairs of $i_{1}, i_{2} \in \mathcal{C}$.

Proof: Suppose $i_{2}=\arg \max _{i \backslash\left\{i_{1}\right\}}\left(\theta_{i} h_{i, j} / \lambda_{i_{1}, i}\right)$. Under the previous cell-selection rule of Eq. (1), the condition under which user $j$ 's serving cell is changed from $i_{1}$ to $i_{2}$ is $\theta_{i_{1}} h_{i_{1}, j}<\theta_{i_{2}} h_{i_{2}, j} / \lambda_{i_{1}, i_{2}}$. Under the new rule of Eq. (6), the condition changes to $\theta_{i_{1}} h_{i_{1}, j} / \lambda_{i_{1}}<\theta_{i_{2}} h_{i_{2}, j} / \lambda_{i_{2}}$. Decisions based on both of the rules are consistent if and only if $\lambda_{i_{1}, i_{2}}=\frac{\lambda_{i_{2}}}{\lambda_{i_{1}}}$.

Proposition 2 implies that the number of offset parameters to determine in $P 1$ is reduced from $C(C-1)$ to $C$. Note that the result of Proposition 2 is compatible with that of Proposition 1.

\section{Control Algorithms}

We first present an assumed radio resource-control algorithm. We then develop range-control algorithms. We also analyze the convergence behavior when these algorithms are combined.

\section{A. Radio Resource Control}

We assume radio resources in each cell are allocated non-cooperatively. The authors of [8] showed that a noncooperative game of sum-rate-maximizing power allocation reaches a Nash equilibrium. In [19], we extended the result to a sum-rate-maximizing RB and power allocation game in twotier femtocell networks by relaxing the RB allocation indicator as a real variable in $[0,1]$. However, sum-rate maximization is not the only operation scenario of a cell since, in many cases, users do not consume more resources than they need due to their application-specific traffic demand and limited data plan. Thus, we focus on a non-cooperative load minimization game with per-user rate constraints and show that it also reaches a Nash equilibrium.

For a given $\vec{\lambda}$, we can separate DL and UL resource allocations, which can be obtained using an analogous procedure. So, we mainly focus on the DL case in this subsection. We define user $j$ 's resource usage rate as $a_{j} \triangleq \sum_{k \in \mathcal{K}} a_{j, k}\left(b_{j}\right.$ for UL). Then, considering the assumption of the even distribution of user's resource usage in frequency and time domains, the DL resource allocation problem of cell $i$ is formulated as

Definition 2 (Cell-load minimization problem in $D L$ ):

$$
P 2: \min _{\vec{x}_{i}} \rho_{i, D L}\left(\vec{x}_{i}\right)=\min _{\vec{x}_{i}}\left(\sum_{j \in U_{i}} a_{j}+\sum_{j \in U_{i}} \frac{a_{j}}{|\mathcal{K}|} \sum_{k \in \mathcal{K}} \omega_{j, k} p_{j, k}\right)
$$

subject to

$C 2.1: 0 \leq a_{j} \leq|\mathcal{K}|$

$C 2.2: 0 \leq p_{j, k} \leq P_{\max }$

$C 2.3: a_{j} B \log _{2}\left(1+\gamma_{j} / \Omega\right)=r_{j}$. 
In $C 2.3, \log _{2}\left(1+\gamma_{j} / \Omega\right)$ is the spectral efficiency. Once $a_{j}$ is determined, the minimum spectral efficiency meeting the constraint is obtained as well. We say that, if the achieved spectral efficiency is equal to or greater than this minimum for all RBs, the user's rate constraint is met sufficiently.

Proposition 3: The non-cooperative cell-load minimization game $P 2$ has a Nash equilibrium.

Proof: From [20], the solution of a non-cooperative utility-maximization game is a Nash equilibrium if (1) the solution space is a nonempty, convex, and compact subset of some Euclidean space; (2) each utility function is continuous and quasi-concave. It is straightforward to show that $P 2$ meets the first condition. We can rewrite $P 2$ as $\min \rho_{i}=\max -\rho_{i}$, so $-\rho_{i}$ should be quasi-concave. Since every convex function is quasi-convex, $\rho_{i}$ is quasi-convex. Then, its negative is quasiconcave.

We first assume that, in the equilibrium, $p_{j, k}$ meets the target SINR $\gamma_{j}$ for all $k$ (otherwise it is saturated with $P_{\max }$ ). That is, we can substitute $p_{j, k}$ in Eq. (7) with $\gamma_{j} h_{i, j}^{-1}(k)\left(I_{j, k}(\vec{p})+\sigma_{j, k}\right)$ where $h_{i, j}(k)$ is the channel gain at RB $k, I_{j, k}(\vec{p})$ is the interference experienced by user $j$ and $\sigma_{j, k}$ is the thermal noise. From the per-user rate constraint, we have $\gamma_{j}=\Omega\left(e^{\mu_{j} / a_{j}}-1\right)$ where $\mu_{j} \triangleq r_{j}(\ln 2) / B$. By substituting $\gamma_{j}$ with this again, the load function of cell $i$ becomes

$$
\rho_{i, D L}\left(\vec{x}_{i}\right)=\sum_{j \in U_{i}} a_{j}\left(1+\nu_{j} \Omega\left(e^{\mu_{j} / a_{j}}-1\right)\right)
$$

where we define $\nu_{j} \triangleq \sum_{k \in \mathcal{K}} \frac{\omega_{j, k}}{|\mathcal{K}|} \frac{I_{j, k}+\sigma_{j, k}}{h_{i, j}(k)}$. By letting $\frac{\partial \rho_{i}}{\partial a_{j}}=$ 0 , we obtain a minimizer, denoted by $a_{j}^{*}$, as ${ }^{5}$

$$
a_{j}^{*}=\min \left\{\left[\frac{\mu_{j}}{\kappa_{j}+1}\right]^{+},|\mathcal{K}|\right\}
$$

where

$$
\kappa_{j} \triangleq W\left(\frac{e^{-1}\left(1-\nu_{j} \Omega\right)}{\nu_{j} \Omega}\right)
$$

( $W$ is the Lambert $W$ function).

Proposition 4: $\omega_{j, k}=\xi_{j, k} /\left(I_{j, k}(\vec{p})+\sigma_{j, k}\right)$ is a sufficient condition of having constant $a_{j}$ ( $\xi_{j, k}$ is a constant).

If the above holds, we have $\nu_{j}=\sum_{k \in \mathcal{K}} \frac{\xi_{j, k}}{|\mathcal{K}| h_{i, j}(k)}$ and thus $\nu_{j}$ becomes independent of $\vec{p}$, i.e., constant. Intuitively, $\omega_{j, k}=$ $\xi_{j, k} /\left(I_{j, k}(\vec{p})+\sigma_{j, k}\right)$ means pricing the consumption of power resource lower for those users suffering higher interference. We henceforth assume that this is applied for all $j$ and $k$. Then, $a_{j}^{*}$ remains constant unless user $j$ 's serving cell is changed by a range control.

From the rate constraint and Eq. (9), we obtain $p_{j, k}$ in the equilibrium, denoted by $p_{j, k}^{*}$ (and its vector form by $\vec{p}^{*}$ ), as:

$$
p_{j, k}^{*}=\min \left\{\frac{I_{j, k}\left(\vec{p}^{*}\right)+\sigma_{j, k}}{h_{i, j}(k)} \Omega\left[e^{\kappa_{j}+1}-1\right]^{+}, P_{\max }\right\}
$$

${ }^{5}[z]^{+}=\max \{z, 0\}$. and this can be rewritten in an iterative form as:

$$
\begin{aligned}
p_{j, k}(t+1) & \triangleq f\left(p_{j, k}(t)\right) \\
& =\min \left\{\frac{p_{j, k}(t)}{\gamma_{j, k}(t)} \Omega\left[e^{\kappa_{j}+1}-1\right]^{+}, P_{\max }\right\}
\end{aligned}
$$

where we call $f\left(p_{k}(t)\right)$ the $D L$ power update function; $\gamma_{j, k}(t)$ is the achieved SINR at $t . f$ is defined for vector input and output as well, i.e., $\vec{p}(t+1)=f(\vec{p}(t))$. The UL power update function, denoted by $g$, is defined similarly.

Proposition 5: The power update functions $f$ and $g$ converge to a unique fixed point.

Proof: Yates [21] showed that an iteration of $\vec{p}(t+1)=$ $f(\vec{p}(t))$ converges to a unique fixed point if it meets (1) positivity: $f(\vec{p}) \succeq 0$; (2) monotonicity: $\vec{p}_{1} \succeq \vec{p}_{2} \rightarrow f\left(\vec{p}_{1}\right) \succeq f\left(\vec{p}_{2}\right)$; and (3) scalability: $\alpha f(\vec{p}) \succeq f(\alpha \vec{p})$ for $\forall \alpha>1$. Since Eq. (12) meets all these conditions, it converges to a unique fixed point, i.e., a Nash equilibrium $\vec{p}^{*}$ given in Eq. (11). We omit the details.

\section{B. Per-Cell Range Control}

1) Baseline: At each iteration, a macrocell first calculates the expected resource allocation when a specific $\vec{\lambda}$ is chosen. If $\vec{\lambda}$ is updated, users' serving cells may be changed and the radio resource controllers will update $\vec{x}$ and $\vec{y}$ accordingly; in particular, a user, if his serving cell is changed, experiences new channel gain and interference. We denote $\vec{x}(\vec{\lambda})=\left(\vec{a}(\vec{\lambda}), f^{(\vec{\lambda})}(\vec{p})\right)$ and $\vec{y}(\vec{\lambda})=\left(\vec{b}(\vec{\lambda}), g^{(\vec{\lambda})}(\vec{q})\right)$. Then, the next range parameter can be simply selected as

$$
\vec{\lambda}^{*}=\arg \min _{\vec{\lambda}} \rho(\vec{x}(\vec{\lambda}), \vec{y}(\vec{\lambda})) .
$$

The size of search space for the above problem is $C \times|\Lambda|$. Since the size for the centralized per-user association problem is $|U| \times C(|U|$ is the number of users), the size of the percell range control is reduced. In general, $|\Lambda|$ is expected to be much smaller than $|U|$.

Allowing simultaneous range updates of all hotzone cells at each iteration as above may speed up convergence, but trigger unnecessary updates exceeding the deactivation threshold. In order to reach the threshold with minimum range updates, we need to minimize control granularity, i.e., allowing only a single hotzone cell to change its range at each iteration. The modified algorithm first checks a maximum achievable decrease of the network load when the range of each cell is adjusted, as shown below:

$$
\lambda_{i}^{*}=\arg \max _{\lambda_{i}}\left\{\Delta^{\left(\vec{\lambda}\left(i: \lambda_{i}\right)\right)}(\vec{x}, \vec{y})\right\}
$$

where

$$
\Delta^{(\vec{\lambda})}(\vec{x}, \vec{y}) \triangleq \rho(\vec{x}, \vec{y})-\rho(\vec{x}(\vec{\lambda}), \vec{y}(\vec{\lambda})) .
$$

Here, $\vec{\lambda}\left(i: \lambda_{i}\right)$ is the range offset vector having the element of cell $i$ changed to $\lambda_{i}$ and the others intact, and $\vec{x}$ and $\vec{y}$ are for the current serving cells. Then, a cell to change to is selected as

$$
i^{*}=\arg \max _{i}\left\{\Delta^{\left(\vec{\lambda}\left(i: \lambda_{i}^{*}\right)\right)}(\vec{x}, \vec{y})\right\} .
$$


That is, a cell yielding the largest gain via range control is chosen first. The range of the selected cell is set to $\lambda_{i^{*}}^{*}$.

Let $T$ be the set of time instants when a range control is made by the range controller. As mentioned earlier, the range controller is activated only when needed. We also assume that, when activated, the range controller adjusts ranges at intervals longer than those of the radio resource controller due to the accompanying cell-switching overhead. Therefore, if the time instants when a radio resource control is made are indexed by consecutive non-negative integers, we have $T \subset\{0,1, \cdots\}$. Finally, the offset parameters and radio resource allocations are updated as:

$$
\begin{aligned}
& \vec{\lambda}(t+1)= \begin{cases}\vec{\lambda}\left(i^{*}: \lambda_{i^{*}}^{*}\right) & t \in T \\
\vec{\lambda}(t) & \text { otherwise }\end{cases} \\
& \vec{x}(t+1)= \begin{cases}\left(\vec{a}(\vec{\lambda}(t+1)), f^{(\vec{\lambda}(t+1))}(\vec{p}(t))\right) & t \in T \\
(\vec{a}(t), f(\vec{p}(t))) & \text { otherwise }\end{cases} \\
& \vec{y}(t+1)= \begin{cases}\left(\vec{b}(\vec{\lambda}(t+1)), g^{(\vec{\lambda}(t+1))}(\vec{q}(t))\right) & t \in T \\
(\vec{b}(t), g(\vec{q}(t))) & \text { otherwise }\end{cases}
\end{aligned}
$$

Once $\vec{\lambda}(t)$ meets $\Delta^{(\vec{\lambda}(t))}(\vec{x}, \vec{y})=0$, the algorithm computes Eq. (13) and checks if the result produces lower $\rho$; if so, $\vec{\lambda}$ is replaced by this result to avoid staying in a local solution; otherwise, the algorithm stops running.

2) Convergence Analysis: We now show that the per-cell range-control algorithm converges.

Proposition 6: The fixed point $\rho^{*}$ is unique and meets $0<$ $\rho^{*} \leq \rho(\vec{x}(\vec{\lambda}), \vec{y}(\vec{\lambda}))$ for $\forall \vec{\lambda}$.

Proof: Suppose that there exists another fixed point $\rho^{\prime}$ smaller than $\rho^{*}$. Let $\left(\vec{x}^{\prime}, \vec{y}^{\prime}\right)$ and $\left(\vec{x}^{*}, \vec{y}^{*}\right)$ be the resource allocation vectors for $\rho^{\prime}$ and $\rho^{*}$, respectively. Since $\rho^{\prime} \neq \rho^{*}$ and the load function is monotonic, we should have $\left(\vec{x}^{\prime}, \vec{y}^{\prime}\right) \neq$ $\left(\vec{x}^{*}, \vec{y}^{*}\right)$. For a fixed $\vec{\lambda}$, the existence of a unique fixed point of $(\vec{x}, \vec{y})$ can be readily proved by Propositions 4 and 5 . Therefore, $\vec{\lambda}^{\prime} \neq \vec{\lambda}^{*}$. Then, $\vec{\lambda}^{*}$ does not meet Eq. (13), which is a contradiction.

The fixed points of $\vec{x}$ and $\vec{y}$ are always larger than 0 due to the non-zero term of the thermal noise $\vec{\sigma}$. Thus, $\rho^{*}>0$.

Proposition 7: Starting from any $\vec{\lambda}$ meeting per-user rate constraints sufficiently, the per-cell range-control algorithm converges to the unique fixed point $\rho^{*}$.

Proof: We first consider that $\vec{\lambda}$ is fixed. If user $j$ 's rate constraint is met at $t$ sufficiently, his current SINR $\gamma_{j, k}(t)$ should be equal to, or greater than the minimum SINR $\gamma_{j}$ that exactly achieves $r_{j}$ as:

$$
\gamma_{j, k}(t) \geq \gamma_{j}=\Omega\left(e^{\mu_{j} / a_{j}^{*}+1}-1\right)=\Omega\left(e^{\kappa_{j}+1}-1\right) .
$$

By multiplying both sides by $p_{j, k}(t) / \gamma_{j, k}(t)$ and referring to Eq. (12), we have

$$
p_{j, k}(t) \geq \frac{p_{j, k}(t)}{\gamma_{j, k}(t)} \Omega\left(e^{\kappa_{j}+1}-1\right)=p_{j, k}(t+1) .
$$

Since $f$ is monotonic as proved in Proposition 5, $f\left(p_{j, k}(t)\right) \geq$ $f\left(p_{j, k}(t+1)\right)$, which yields $p_{j, k}(t+1) \geq p_{j, k}(t+2)$. That is, $\vec{p}(t)$ decreases monotonically, and so does $\vec{q}(t)$. Therefore, $\rho(\vec{x}(0), \vec{y}(0)) \geq \cdots \geq \rho(\vec{x}(t), \vec{y}(t))$ holds due to the monotonicity of the load function. In what follows, we show that this also holds when range control is performed together.

Eq. (14) can be rewritten as

$$
\begin{aligned}
& \max _{\lambda_{i}}\left\{\Delta^{\left(\vec{\lambda}\left(i: \lambda_{i}\right)\right)}(\vec{x}, \vec{y})\right\} \\
& =\rho(\vec{x}, \vec{y})-\min _{\lambda_{i}} \rho\left(\vec{x}\left(\vec{\lambda}\left(i: \lambda_{i}\right)\right), \vec{y}\left(\vec{\lambda}\left(i: \lambda_{i}\right)\right)\right) .
\end{aligned}
$$

That is, the algorithm minimizes $\rho\left(\vec{x}\left(\vec{\lambda}\left(i: \lambda_{i}\right)\right), \vec{y}(\vec{\lambda}(i \quad\right.$ : $\left.\left.\left.\lambda_{i}\right)\right)\right)$ and thus $\rho\left(\vec{x}\left(\vec{\lambda}\left(i: \lambda_{i}^{*}\right)\right), \vec{y}\left(\vec{\lambda}\left(i: \lambda_{i}^{*}\right)\right)\right) \leq \rho(\vec{x}(\vec{\lambda}(i$ : $\left.\left.\left.\lambda_{i}\right)\right), \vec{y}\left(\vec{\lambda}\left(i: \lambda_{i}\right)\right)\right)$. Therefore, when $t \in T$,

$$
\begin{aligned}
\rho(\vec{x}(t+1), \vec{y}(t+1)) & =\rho\left(\vec{x}\left(\vec{\lambda}\left(i^{*}: \lambda_{i^{*}}^{*}\right)\right), \vec{y}\left(\vec{\lambda}\left(i^{*}: \lambda_{i^{*}}^{*}\right)\right)\right) \\
& \leq \rho\left(\vec{x}\left(\vec{\lambda}\left(i: \lambda_{i}^{*}\right)\right), \vec{y}\left(\vec{\lambda}\left(i: \lambda_{i}^{*}\right)\right)\right) \\
& \leq \rho(\vec{x}(\vec{\lambda}(t)), \vec{y}(\vec{\lambda}(t))) \\
& =\rho(\vec{x}(t), \vec{y}(t))
\end{aligned}
$$

which implies that the network load decreases monotonically as the algorithm goes through iterations. Since the sequence of $\rho(\vec{x}(t), \vec{y}(t))$ is monotonically decreasing and bounded below by $\rho^{*}$, it converges to $\rho^{*}$.

\section{Universal Range Control}

To reduce the search space further, we consider mapping a universal parameter to the offset parameters of cells instead of calculating each of them. First, we define user's load contribution which will be used in the sequel.

Definition 3 (User's load contribution): User $j$ 's contribution of load to cell $i$, denoted by $\Delta \rho_{i, j}$, is the increase of cell $i$ 's load when user $j$ is connected to it while other users remain connected to their current serving cells.

Below we explain the mapping rule of the given information to cells' offset parameters.

Proposition 8: A sufficient condition of the offset parameters getting a user assigned to a cell resulting in his minimal load contribution is given as

$$
\lambda_{i}=\frac{h_{i, j} \theta_{i} \Delta \rho_{i, j}}{h_{0, j} \theta_{0} \Delta \rho_{0, j}} \varphi
$$

where $\varphi$ is a network-wide control variable.

Proof: Consider two candidate cells $i_{1}$ and $i_{2}$ for user $j$ to connect to. If user $j$ gets connected to one of them, his radio resource allocation is updated accordingly. Then, for user $j$ to minimize his load contribution, the target cell, denoted by $i^{*}$, should be chosen such that $i^{*}=\arg \min _{i} \Delta \rho_{i, j}$. Then, the problem is reduced to that of choosing the one minimizing $\Delta \rho_{i, j}$. If $\Delta \rho_{i_{1}, j} / \Delta \rho_{i_{2}, j} \geq 1$, it is beneficial to choose $i_{2}$, else $i_{1}$. On the other hand, as shown in Eq. (6), $h_{i_{1}, j} \theta_{i_{1}} \lambda_{i_{2}} / h_{i_{2}, j} \theta_{i_{2}} \lambda_{i_{1}} \geq 1$ leads to handing user $j$ over to cell $i_{1}$. Therefore, to make consistent decisions, we can relate $\Delta \rho_{i, j}$ and $\lambda_{i}$ as $\Delta \rho_{i_{1}, j} / \Delta \rho_{i_{2}, j}=h_{i_{2}, j} \theta_{i_{2}} \lambda_{i_{1}} / h_{i_{1}, j} \theta_{i_{1}} \lambda_{i_{2}}$. Consequently, we can obtain Eq. (21) where the denominator is given by a reference cell (e.g., macrocell).

Note that the offset parameter obtained in Eq. (21) is userspecific due to $h_{i, j}$ and $\Delta \rho_{i, j}$. In order to obtain a cellwide offset parameter, we need to eliminate these user-specific terms. For analytical tractability, we restrict our concern to 
UL power resource-limited HetNets (i.e., $\rho_{i}=\rho_{i, U L}=$ $\left.\sum_{j \in U_{i}} b_{j} \sum_{k} q_{j, k}\right)$ with users having a fixed target SINR.

Proposition 9: Assume channel reciprocity and a fixed target SINR. When only a UL power load is considered in the range control, a cell-wide offset parameter leading to minimal load contribution of each user is obtained as

$$
\lambda_{i}=\frac{\theta_{i}\left(\sum_{k \in \mathcal{K}} J_{k}^{(i)}+\sigma_{i}\right)}{\theta_{0}\left(\sum_{k \in \mathcal{K}} J_{k}^{(0)}+\sigma_{0}\right)} \varphi
$$

where $J_{k}^{(i)}$ is the level of UL interference experienced by cell $i$ at RB $k$ and $\sigma_{i}$ is the thermal noise of cell $i$.

Proof: Let $b_{j}(i)$ and $q_{j, k}(i)$ denote the UL resource allocation of user $j$ when he is served by cell $i$. Since we consider a UL power load only, Eq. (21) is rewritten as:

$$
\begin{aligned}
\lambda_{i} & =\frac{h_{i, j} \theta_{i} \sum_{k \in \mathcal{K}} b_{j}(i) q_{j, k}(i)}{h_{0, j} \theta_{0} \sum_{k \in \mathcal{K}} b_{j}(0) q_{j, k}(0)} \varphi \\
& =\frac{h_{i, j} \theta_{i} b_{j}(i) \delta_{j} \sum_{k \in \mathcal{K}}\left(J_{j, k}^{(i)}(\vec{q})+\sigma_{i, k}\right) h_{j, i}^{-1}(k)}{h_{0, j} \theta_{0} b_{j}(0) \delta_{j} \sum_{k \in \mathcal{K}}\left(J_{j, k}^{(0)}(\vec{q})+\sigma_{i, k}\right) h_{j, 0}^{-1}(k)} \varphi \\
& =\frac{h_{i, j} \theta_{i} \sum_{k \in \mathcal{K}}\left(J_{j, k}^{(i)}(\vec{q})+\sigma_{i, k}\right) h_{j, i}^{-1}(k)}{h_{0, j} \theta_{0} \sum_{k \in \mathcal{K}}\left(J_{j, k}^{(0)}(\vec{q})+\sigma_{i, k}\right) h_{j, 0}^{-1}(k)} \varphi
\end{aligned}
$$

where the second equality follows from the fact that, for a fixed $\delta_{j}, b_{j}(i)$ is fixed as well (as can be seen in the per-user rate constraint). We assume that users connected to the same cell experience identical UL interference due to randomized inter-cell interferences, i.e., $J_{j, k}^{(i)} \approx J_{k}^{(i)}$ for $\forall j \in U_{i}$. We also assume that the channel gain at each RB has an identical distribution and thus, on average, $h_{j, i}(k) \approx h_{j, i}$. We can then approximate $\lambda_{i}$ as

$$
\begin{aligned}
\lambda_{i} & \approx \frac{h_{i, j} \theta_{i} \sum_{k \in \mathcal{K}}\left(J_{k}^{(i)}+\sigma_{i, k}\right) h_{j, i}^{-1}}{h_{0, j} \theta_{0} \sum_{k \in \mathcal{K}}\left(J_{k}^{(0)}+\sigma_{i, k}\right) h_{j, 0}^{-1}} \varphi \\
& =\frac{\theta_{i}\left(\sum_{k \in \mathcal{K}} J_{k}^{(i)}+\sigma_{i}\right)}{\theta_{0}\left(\sum_{k \in \mathcal{K}} J_{k}^{(0)}+\sigma_{0}\right)} \varphi
\end{aligned}
$$

where the second equality follows from the assumed channel reciprocity.

Proposition 9 implies that a hotzone cell experiencing higher interference will have a smaller range. Consequently, under the universal range-control algorithm, the range of each hotzone cell is obtained immediately, provided that the pilot transmit power, UL interference level and thermal noise of a reference cell are given. It is noted that the pilot transmit power and the thermal noise are typically fixed. Moreover, if the master controller is operated by a reference cell, it can obtain the radio resource status of each cell from the reported offset values based on Eq. (22). Thus hotzone cells don't need to report the current resource status to the master controller separately. Therefore, the universal range-control algorithm can be implemented in a fully distributed manner with a greatly reduced inter-cell signaling overhead compared to the per-cell range control. $\varphi$ can be controlled to adjust a load balance between macro and hotzone cells.
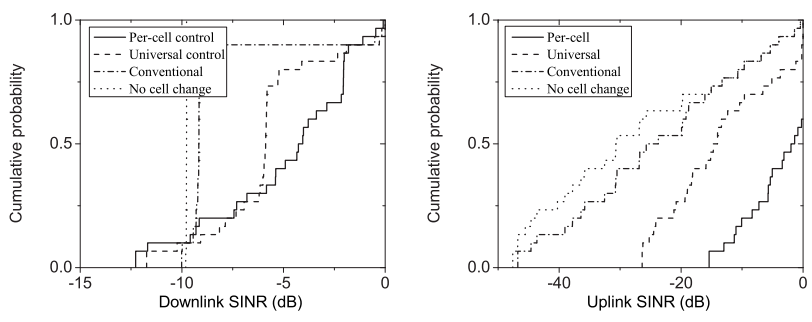

(a) Various cell selection mechanisms
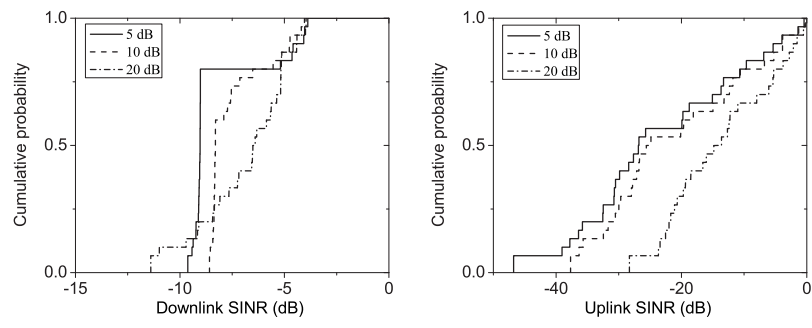

(b) Fixed cell ranges

Fig. 2. Probability distribution of achieved DL and UL SINRs for different control mechanisms (top) and fixed cell ranges (bottom) (the numbers of users and hotzone cells are fixed at 30 and 20 , respectively)

\section{Evaluation}

To evaluate the effectiveness of ARCHoN, we consider a single-sector macrocell on which multiple outdoor hotzone cells are overlaid. We follow the simulation scenario of 3GPP [2] for hotzone deployment and path-loss models. The radius of a macrocell is $500 \mathrm{~m}$ and users are randomly placed in the macrocell; the angle and the distance of each to the macro BS are randomly chosen with a uniform probability distribution. Hotzone BSs are also randomly distributed within a macrocell. The minimum distance between a user and the macro BS and that between a hotzone BS and the macro BS are set to $35 \mathrm{~m}$ and $75 \mathrm{~m}$, respectively. The path losses between users and BSs are determined as:

- macro BS $\leftrightarrow$ user: $h=128.1+37.6 \log _{10} d$;

- hotzone BS $\leftrightarrow$ user: $h=140.7+36.7 \log _{10} d$,

where $d$ is the transmitter-receiver separation distance in kilometers. We also assume the log-normal shadowing with a standard deviation of $8 \mathrm{~dB}$.

Both macro and hotzone BSs operate at the frequency band of $2 \mathrm{GHz}$ with the channel bandwidth of $5 \mathrm{MHz}$. A slot is defined as one unit of time, and each slot is set to $1 \mathrm{~ms}$. RB and transmit power allocation changes at a slot-time scale. The maximum transmit powers of the macro and hotzone BSs are 40 and $30 \mathrm{dBm}$, respectively; the antenna pattern of BSs is omnidirectional and the antenna gain plus a connector loss is 5 $\mathrm{dBi}$. The minimum and maximum transmit powers inherently given to user devices are assumed to be -50 and $23 \mathrm{dBm}$, respectively. The target date rate of users is set to $1 \mathrm{Mbps}$ for both DL and UL. The range controller makes control decisions once every 100 slots; the offset parameter is selected within the set $\Lambda=\{0 \mathrm{~dB},-1 \mathrm{~dB}, \ldots,-20 \mathrm{~dB}\}$. For the universal range control, $\varphi$ is set to one. 

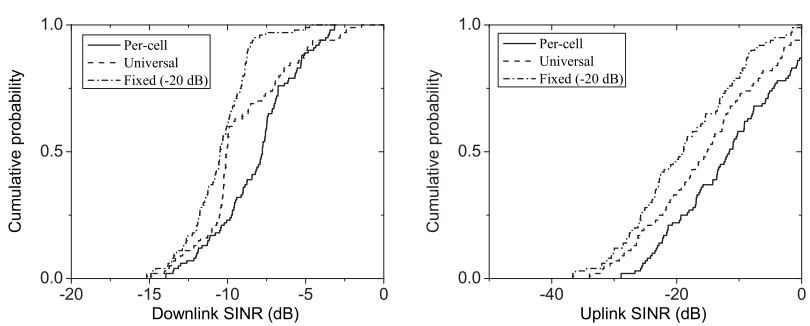

Fig. 3. Probability distribution of achieved DL (left) and UL (right) SINRs for adaptive and fixed range control mechanisms under non-uniform user distribution (the numbers of users and hotzone cells are fixed at 100 and 20 , respectively)

The achieved SINRs of different control mechanisms are compared in Fig. 2 for DL (left) and UL (right). First, it is shown that the conventional cell selection based on the strongest received power achieves better SINRs of users than the case without allowing connections to hotzone cells. This result clearly shows the benefit of HetNets since even a nonoptimal control of cell selection improves performance. Such an improvement comes from (1) reduced loss of paths to serving cells and (2) macrocell offloading. ARCHoN with either per-cell or universal range control, as expected from the ability to reduce interference, further improves the achieved SINRs of users by making cell-change decisions towards the minimum network load. Meanwhile, the bottom figures consider the fixed cell-range case where all hotzone cells have the same offset value. As the offset parameter decreases, the performance gets better; when the offset value is $-20 \mathrm{~dB}$, the achieved SINRs are close to those of the universal range control (the offset value lower than $-20 \mathrm{~dB}$ showed almost no change or slight performance degradation). However, this result does not mean that large and identical fixed ranges for all hotzone cells can be a desirable solution. To prove this, we compare ARCHoN with the fixed range case under a nonuniform user distribution in Fig. 3; half of users are randomly distributed within $50 \mathrm{~m}$ from hotzone cells while keeping the other half of users distributed as before. The first half of users select randomly hotzone cells among half of the all. The non-uniform user distribution scenario is acceptable in the real world since hotzone cells will typically be deployed to cover hotspot areas. The figure shows that the universal range control achieves better performance than the fixed range case with the minimum offset value (thus with the largest range) in both DL and UL. This implies that ARCHoN improves the performance of a HetNet by adaptively adjusting cell ranges based on cells' conditions and balancing cell loads properly, instead of simply extending cell ranges to maximum. The percell range-control algorithm still shows the best performance among those considered.

Next, the effects of the number of users $(|U|)$ and the number of hotzone cells $(S)$ are explored in Figs. 4 and 5, respectively. The results show a common trend that the increasing number of users deteriorates the SINRs of all users for both per-cell and universal range controls due to the increase
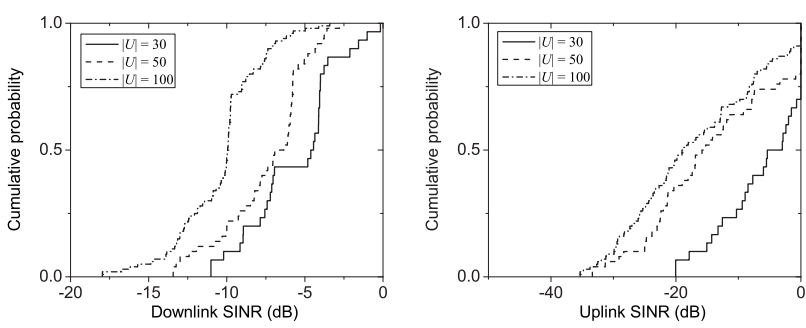

(a) Per-cell range control
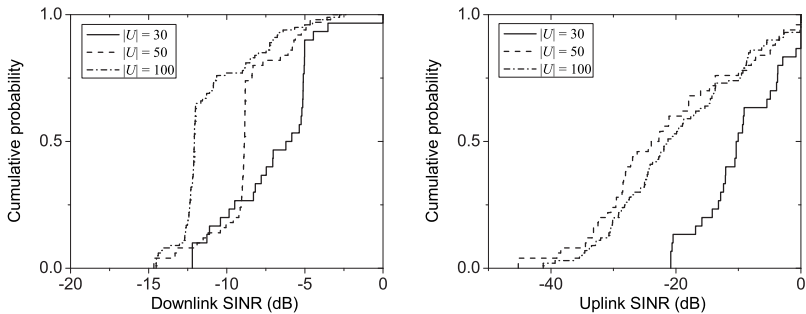

(b) Universal range control

Fig. 4. Probability distribution of users' achieved SINR for DL and UL under various number of users when range control is considered (the number of hotzone cells is fixed at 10)
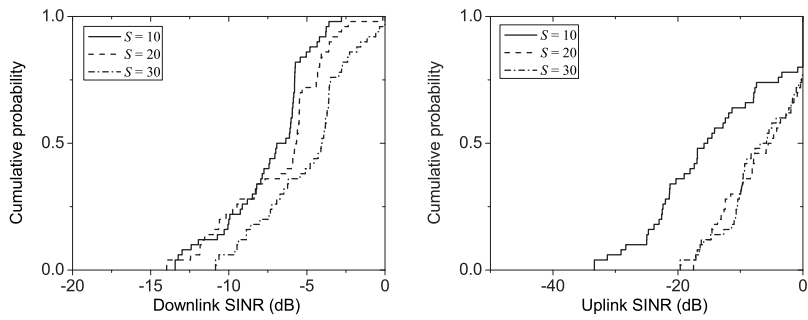

(a) Per-cell range control
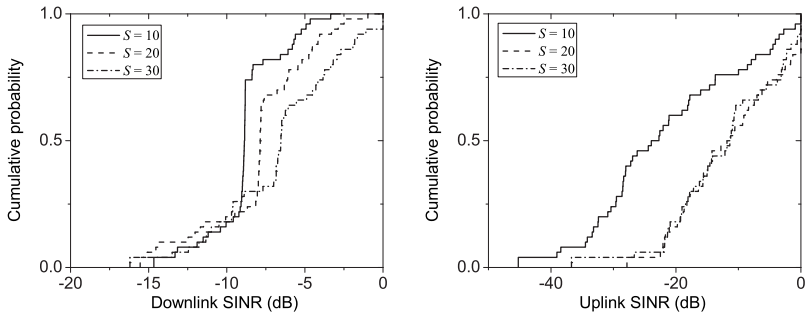

(a) Universal range control

Fig. 5. Probability distribution of users' achieved SINR for DL and UL under various number of hotzone cells when range control is considered (the number of users is fixed at 50)

in the number of interference sources. However, an opposite effect of the number of hotzone cells is observed; as the number of hotzone cells increases, the users' achieved SINRs are improved significantly. This is because users have more opportunities to connect to local hotzone cells with smaller path losses; those users will be allocated lower transmit powers for both DL and UL, thus producing fewer interferences to others. However, increasing the number of hotzone cells does not always improve the performance; when $S$ is increased from 20 to 30 , the UL SINRs of users remain almost intact under 

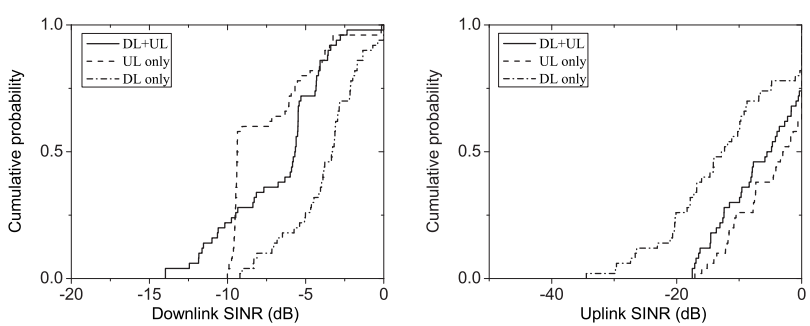

Fig. 6. Probability distribution of users' achieved SINR under various load definitions (the numbers of users and hotzone cells are fixed at 50 and 20, respectively)

both control algorithms. Therefore, the number of hotzone cells to deploy in HetNets should be determined by carefully considering various factors. Meanwhile, it is observed that in the DL case, the users having low SINRs are not influenced by the increased number of hotzone cells; most of them are located at the boundary of the macrocell and don't have hotzone cells in their neighborhood. Those users are strongly interfered by other users, but have insufficient room to increase their transmit power to combat the interference.

Finally, we study the effect of the load function. We consider three different load functions for the per-cell range control: (1) the sum of both DL and UL resource consumptions (DL+UL); (2) the sum of DL resource consumptions only (DL-only); and (3) the sum of UL resource consumptions only (UL-only). The corresponding simulation results are plotted in Fig. 6 when $|U|$ $=50$ and $S=20$. We can observe that, when only the DL resource consumptions are considered in the load, users achieve higher DL SINRs, but lower UL SINRs. This is because, under such a load definition, the network will be operated such that DL resource consumptions of all cells are minimized and thus DL interferences are reduced. A similar trend is observed for the UL-only case as well. Meanwhile, the load considering both DL and UL resource consumptions shows a balanced result between the DL-only and UL-only cases. Since a user's communication call typically accompanies both DL and UL transmission and the importance of UL communications is increasing due to emerging interactive and content uploading services, it is desirable to balance the performance of DL and UL by considering both of them in the load.

\section{CONCLUSION}

In this paper, we have presented a joint radio resource and cell range-control framework, called ARCHoN, for HetNets. ARCHoN is composed of three complementary controllersmaster, range, and radio-resource controllers-which activate/deactivate the range controller, make range-control decisions, and handle radio resource scheduling. ARCHoN activates the range controller only when needed and finds a control sequence to minimize the inter-cell signaling while reaching a desired system condition. The range controller iteratively controls hotzone cells' ranges to minimize the defined load; range-control decisions are made by either per-cell or universal control algorithms which differ in performance, computation complexity, and signaling overhead. The radio resource controller derives a sequence of frequency, time and power resource allocations reaching a unique Nash equilibrium. ARCHoN successfully overcomes the performance limit of the existing cell-selection framework and achieves better performance than simple range extension. In addition, it does not require any modification of user devices or an air interface, and can thus be applicable to real systems.

\section{ACKNOWLEDGEMENT}

The work reported in this paper was supported in part by the National Science Foundation under Grant CNS-1160775.

\section{REFERENCES}

[1] S.-P. Yeh, S. Talwar, G. Wu, N. Himayat, and K. Johnsson, "Capacity and coverage enhancement in heterogeneous networks," IEEE Wireless Communications, vol. 18, no. 3, pp. 32 -38, June 2011.

[2] Further advancements for E-UTRA physical layer aspects, 3GPP Technical Report 36.814, Rev. 9.0.0, Mar. 2010.

[3] Motorola, On Range Extension in Open-access Heterogeneous Networks, 3GPP Technical contribution R1-103 181, May 2010.

[4] Samsung, System Performance of Heterogeneous Networks with Range Expansion, 3GPP Technical contribution R1-100 142, Jan. 2010.

[5] Y. Bejerano and S.-J. Han, "Cell breathing techniques for load balancing in wireless LANs," IEEE Transactions on Mobile Computing, vol. 8, no. 6, pp. $735-749$, June 2009.

[6] W. Yu and R. Lui, "Dual methods for nonconvex spectrum optimization of multicarrier systems," IEEE Transactions on Communications, vol. 54, no. 7, pp. $1310-1322$, July 2006.

[7] Z. Shen, J. G. Andrews, and B. L. Evans, "Adaptive resource allocation in multiuser OFDM systems with proportional rate constraints," IEEE Transactions on Wireless Communications, vol. 4, no. 6, pp. 2726-2737, 2005.

[8] H. Kwon and B. G. Lee, "Distributed resource allocation through noncooperative game approach in multi-cell OFDMA systems," in Proc. IEEE Int. Conf. Communications ICC '06, vol. 9, 2006, pp. 4345-4350.

[9] A. Stolyar and H. Viswanathan, "Self-organizing dynamic fractional frequency reuse for best-effort traffic through distributed inter-cell coordination," in IEEE INFOCOM 2009, Apr. 2009, pp. 1287 -1295.

[10] K. W. Choi, E. Hossain, and D. I. Kim, "Downlink subchannel and power allocation in multi-cell OFDMA cognitive radio networks," IEEE Transactions on Wireless Communications, vol. 10, no. 7, pp. 2259 2271, July 2011.

[11] R. D. Yates and C.-Y. Huang, "Integrated power control and base station assignment," IEEE Transactions on Vehicular Technology, vol. 44, no. 3, pp. 638-644, 1995.

[12] J.-W. Lee, R. R. Mazumdar, and N. B. Shroff, "Joint resource allocation and base-station assignment for the downlink in CDMA networks," IEEE/ACM Transactions on Networking, vol. 14, no. 1, pp. 1-14, 2006.

[13] L. Smolyar, I. Bergel, and H. Messer, "Unified approach to joint power allocation and base assignment in nonorthogonal networks," IEEE Transactions on Vehicular Technology, vol. 58, no. 8, pp. 4576-4586, 2009.

[14] H. Kim, G. de Veciana, X. Yang, and M. Venkatachalam, " $\alpha$-optimal user association and cell load balancing in wireless networks," in IEEE INFOCOM 2010, Mar. 2010, pp. 1 -5.

[15] Evolved Universal Terrestrial Radio Access Network (E-UTRAN); X2 general aspects and principles, 3GPP Technical Specification 36.420, Rev. 10.1.0, June 2011.

[16] Evolved Universal Terrestrial Radio Access (E-UTRA); Physical Channels and Modulation, 3GPP Technical Specification 36.211, Rev. 9.1.0, Mar. 2010.

[17] Evolved Universal Terrestrial Radio Access (E-UTRA) and Evolved Universal Terrestrial Radio Access Network (E-UTRAN); Overall description, 3GPP Technical Specification 36.300, Rev. 11.4.0, Dec. 2012.

[18] S. T. Chung and A. J. Goldsmith, "Degrees of freedom in adaptive modulation: a unified view," IEEE Transactions on Communications, vol. 49, no. 9, pp. 1561-1571, 2001.

[19] J.-H. Yun and K. G. Shin, "Adaptive interference management of OFDMA femtocells for co-channel deployment," IEEE Journal on Selected Areas in Communications, vol. 29, no. 6, pp. 1225 -1241, June 2011. 
[20] J. F. Nash, "Equilibrium points in n-person games," in Proc. Natl. Acad. Sci. U.S.A., vol. 36, no. 1, Jan. 1950, pp. 48-49.

[21] R. Yates, "A framework for uplink power control in cellular radio systems," IEEE Journal on Selected Areas in Communications, vol. 13, no. 7, pp. $1341-1347$, Sept. 1995. 